\title{
El cambio climático y el comercio de derechos de emisión en la Unión Europea
}

\author{
Mariano H. Novelli* \\ Universidad Abierta Interamericana, Argentina \\ mnovelli@fder.unr.edu.ar \\ Julieta C. Tabares* \\ Universidad Nacional de Rosario, Argentina \\ julietatabares10@hotmail.com
}

Fecha de recibido: 11 de noviembre de 2013 / Fecha de aprobación: 24 de abril 2014

Resumen

El presente estudio aborda desde una perspectiva compleja la problemática del ambiente, focalizándose en la respuesta jurídica que brinda la Unión Europea ante el cambio climático. A tal fin, se detiene inicialmente en el fenómeno del efecto invernadero, para luego analizar los graves riesgos del cambio climático. Indaga asimismo sobre los instrumentos normativos que rigen en el ámbito internacional, en particular la Convención Marco sobre el Cambio Climático y el Protocolo de Kioto. Finalmente, examina exhaustivamente el Régimen para el comercio de derechos de emisión de gases de efecto invernadero, vigente en la Unión Europea a partir de la Directiva 2003/87, sus normas modificatorias y complementarias. Como conclusión, la investigación afirma que el comercio de derechos de emisión constituye la herramienta fundamental con que cuenta el bloque integrado para mitigar los efectos adversos del cambio climático.

\section{Palabras clave}

Cambio climático / Derecho Internacional / comercio de derechos de emisión / Unión Europea

* Profesor de Introducción al Pensamiento Científico en la Facultad de Derecho y Ciencias Políticas de la Universidad Abierta Interamericana (UAI), sede Rosario. Profesor de Derecho Ambiental en la Facultad de Derecho de la Universidad Nacional de Rosario (UNR). Prosecretario en el Poder Judicial de la Provincia de Santa Fe. Coordinador de la Maestría en Derecho Privado de la UNR. Becario Fulbright del Departamento de Estado de los Estados Unidos. Premios "Corte Suprema de Justicia de la Provincia de Santa Fe" y "Corte Suprema de Justicia de la Nación”.

* Docente de Derecho Ambiental en la Facultad de Derecho de la Universidad Nacional de Rosario (UNR). Becaria del Consejo de Investigaciones de la UNR (CIUNR). Auxiliar docente de Derecho Civil III en la Facultad de Derecho de la UNR. Integrante del Centro de Investigaciones de Derecho Civil de la Facultad de Derecho de la UNR. Becaria del Programa AVE Docente en la Facultad de Derecho de la Universidad de Valladolid. 


\section{Deerecho}

\section{Abstract}

This study approaches the problems of the environment from a complex perspective, focusing on the legal response provided by the European Union to climate change. For this purpose, it initially considers the greenhouse effect, and then analyzes the serious risks of climate change. It also inquires about international juridical instruments, in particular the Framework Convention on Climate Change and the Kyoto Protocol. Finally, it thoroughly examines the Scheme for greenhouse gas emission allowance trading, in force in the European Union after the Directive 2003/87, its amendments and complementary norms. In conclusion, the research aseverates that emission allowance trading is the main tool available for the integrated block in order to mitigate the adverse effects of climate change.

\section{Key words}

Climate change / International Law / emission allowance trading / European Union

\section{Tabla de contenido}

1. Introducción 2. Efecto invernadero 2.1. Generalidades 2.2. Gases de efecto invernadero 3. Cambio climático 3.1. Caracterización del fenómeno 3.2. Efectos 4. Cambio climático en el Derecho Internacional 4.1. Convención Marco sobre el Cambio Climático 4.2. Protocolo de Kioto 5. Cambio climático en el Derecho de la Unión Europea 5.1. Instrumentos generales 5.2. El comercio de derechos de emisión 5.2.1. Libro Verde 5.2.2. Régimen para el comercio de derechos de emisión 5.2.2.1. Eje del sistema: Directiva 2003/87 5.2.2.1.1. Conceptos fundamentales 5.2.2.1.2. Permisos de emisión 5.2.2.1.3. Derechos de emisión 5.2.2.1.3.1. Asignación 5.2.2.1.3.2. Entrega 5.2.2.1.4. Mercado de derechos de emisión 5.2.2.1.5. Registro de derechos de emisión 5.2.2.2. Normas complementarias y modificatorias 5.2.2.2.1. Directiva 2004/101 5.2.2.2.2. Directivas 2008/101 y 2009/29 5.2.2.2.3. Reglamentos 219/2009 y 1031/2010 5.2.2.2.4. Decisiones 2006/780 y 2007/589 6. Conclusión. Referencias Bibliográficas 


\section{Introducción}

Los problemas ambientales constituyen hogaño una amenaza sumamente delicada para el género humano, al poner en riesgo la vida animal y vegetal pero sobre todo la continuidad de su propia especie.

Entre las distintas problemáticas que afectan al medio, indubitablemente una de las más acuciantes es la del cambio climático, no sólo por su gravedad intrínseca y actual sino fundamentalmente por los descomunales efectos que puede acarrear. Consecuencias que, por lo demás, resultan difícilmente evaluables con exactitud a priori, pero cuya potencialidad nociva la ciencia tiene absolutamente comprobada.

En este contexto, el presente trabajo se propone analizar inicialmente el cambio climático desde una perspectiva técnica y jurídica, para luego profundizar en uno de los sistemas más interesantes que se han desarrollado a nivel mundial con el objeto de combatirlo, efectuando un detenido estudio del comercio de derechos de emisión en la Unión Europea.

\section{Efecto invernadero}

\subsection{Generalidades}

El sistema climático, según la Convención Marco de las Naciones Unidas sobre el Cambio Climático, está constituido por la totalidad de la atmósfera, la hidrosfera, la biosfera y la geosfera, y sus interacciones. Entre los fenómenos que comprende se encuentra el efecto invernadero, gracias al cual es posible la vida en la Tierra al mantenerse una temperatura media constante.

El proceso se inicia con la llegada de la radiación solar, que atraviesa las nubes y alcanza la superficie terrestre. Sin embargo, no toda la energía del sol es retenida, sino que una parte es emitida nuevamente al espacio en forma infrarroja y absorbida por los denominados "gases de efecto invernadero", calentándose así tanto la superficie como el aire que la rodea (Camps, 2010, pp. 48-49).

Está científicamente acreditado que sin los gases de efecto invernadero, la Tierra sería casi treinta grados más fría que en la actualidad, tornándose de tal modo imposible la existencia humana, animal y vegetal (Martínez Ataz \& Díaz de Mera Morales, 2004, p. 22; Saura Estapa, 2013, p. 13). 


\subsection{Gases de efecto invernadero}

Ciertos gases de efecto invernadero surgen naturalmente pero están influenciados de forma directa o indirecta por las actividades humanas, mientras que otros son totalmente antropogénicos (Aguilar Rojas \& Iza, 2009, p. 409; Cuatecontzi \& Gasca, 2004, p. 87).

Los principales de origen natural son el vapor de agua $(\mathrm{H} 2 \mathrm{O})$, el dióxido de carbono (CO2), el ozono (O3), el metano (CH4) y el óxido nitroso (N2O) ; mientras que los más importantes de carácter antropogénico son los clorofluorocarbonos (CFCs), los hidroclorofluorocarbonos (HCFCs), los hidrofluorocarbonos (HFCs), los perfluorocarbonos (PFCs) y el hexafluoruro de azufre (SF6) .

\section{Cambio climático}

\subsection{Caracterización del fenómeno}

Actualmente, existe consenso científico acerca de que el clima global está sufriendo modificaciones progresivas y constantes. Ello se debe principalmente a la acentuación del efecto invernadero como consecuencia de la acción del hombre. De allí que este fenómeno se conozca como efecto invernadero ampliado o, más frecuentemente, cambio climático.

El proceso ha sido definido por la Convención respectiva como "todo cambio de clima atribuido directa o indirectamente a la actividad humana que altera la composición de la atmósfera mundial y que se suma a la variabilidad natural del clima observada durante períodos de tiempo comparables".

Recientes investigaciones acerca de la temperatura media global muestran el acentuado aceleramiento de su elevación. Los análisis más pesimistas estiman que el calentamiento podría alcanzar entre 4 y $7{ }^{\circ} \mathrm{C}$ para el año 2100 , afectándose así profundamente la civilización humana y los principales ecosistemas del mundo. En contraposición, los estudios más optimistas proyectan que, mediando un urgente recorte en el uso de combustibles fósiles y preservándose activamente los bosques, dicho aumento sería de entre 2 y $3{ }^{\circ} \mathrm{C}$ (Allison, Bindoff, Bindschadler, Cox, de Noblet-Ducoudré, England et al., 2009, p. 51). 


\subsection{Efectos}

Como consecuencia de este proceso, se prevé que se producirán grandes alteraciones ambientales, económicas y sociales. Entre estos efectos, siguiendo a Moreno Sánchez \& Urbina Soria (2008, pp. 26-38), Petit \& Prudent (2008, pp. 129-131) y Torres \& Gómez (2008, pp. 13-19), pueden mencionarse:

- La desaparición de vastas áreas de selvas y bosques debido a la propagación descontrolada de incendios por la sequía.

- El alongamiento de la brecha entre los países desarrollados y aquellos en vías de desarrollo en cuanto a producción y consumo, por la disminución de la productividad en las regiones bajas, situación que aumentaría el riesgo de hambruna para millones de personas.

- El ascenso del nivel del mar debido al derretimiento de los glaciares y casquetes polares.

- La alteración significativa de los ecosistemas marinos por la acidificación oceánica.

- La merma de los cultivos de maíz, trigo, cebada y uva, debido a la reducción de la época de cosecha.

- La intensificación de los ciclones tropicales.

- La propagación de enfermedades transmitidas por vectores y afecciones cardiorrespiratorias a causa del incremento en la concentración de ozono.

- La degradación y salinización de tierras fértiles debido a sucesos meteorológicos como las inundaciones en las masas continentales.

- La extinción de numerosas especies de flora y fauna.

- La reducción en la disponibilidad de agua por el aumento de la evapotranspiración.

Esta serie de cambios, altamente perniciosos para la humanidad, han sido motivadores para la adopción de medidas desde diversas áreas. Seguidamente enunciaremos las principales construcciones jurídicas que se han elaborado en torno a la problemática, tanto a nivel internacional como en el ámbito de la Unión Europea. 


\section{Cambio climático en el Derecho Internacional}

\subsection{Convención Marco sobre el Cambio Climático}

En el Derecho Internacional, la temática del cambio climático cobró relevancia hace más de dos décadas, cuando diversos Estados decidieron impulsar un instrumento jurídico que estableciera los principales lineamientos para la acción. Esto condujo a la negociación y aprobación en 1992 de la Convención Marco de las Naciones Unidas sobre el Cambio Climático, que comprometió a sus firmantes a cumplir la meta de "estabilizar la concentración de gases de invernadero en la atmósfera a niveles que eviten interferencias antrópicas con el sistema climático" (Bellorio Clabot, 2004, p. 197).

No obstante, el texto definitivo de la Convención resultó ser muy amplio, ya que no cuantificó las reducciones de gases de efecto invernadero país por país ni estableció mecanismos concretos para mitigar el cambio climático.

\subsection{Protocolo de Kioto}

La falencia apuntada llevó a que los Estados Partes resolvieran adoptar nuevos criterios para poder cumplir las obligaciones de la Convención, lo cual lograron mediante el Protocolo de Kioto aprobado en 1997, que entró en vigor el 16 de febrero de 2005 (Novelli, 2007, p. 211). Este instrumento ha sido ratificado por 191 países y la Unión Europea. Por su parte, Estados Unidos, el mayor emisor de gases de efecto invernadero, no ha formalizado la ratificación.

E1 Protocolo, a diferencia de la Convención, determinó compromisos cuantificados. La primera etapa de implementación se extendió desde el año 2008 hasta el 2012. Posteriormente fue prorrogado por un segundo período que abarca desde 2013 hasta 2020, merced al consenso alcanzado en la $18^{\text {a }}$ Conferencia de las Partes de la Convención Marco sobre el Cambio Climático (COP 18).

El acuerdo institucionaliza tres tipos de mecanismos complejos para flexibilizar las obligaciones asumidas:

1. Los mecanismos de desarrollo limpio (MDL) se dan entre países desarrollados y países en desarrollo que no tienen la obligación de reducir sus emisiones, cuando uno de los primeros acuerda con uno de los segundos la realización de un proyecto limpio cuyo rédito será un crédito que le permitirá aumentar sus emisiones. 
2. Los mecanismos de aplicación conjunta $(\mathrm{AC})$ consisten en la realización de proyectos en países desarrollados o con economías en transición que generen un ahorro de emisiones adicional al que se hubiera producido en el supuesto de haber empleado tecnología convencional, o no haber incentivado la capacidad de absorción de las masas forestales.

3. El comercio de emisiones permite a los países desarrollados o en transición adquirir créditos de otros de idéntica condición para alcanzar, de forma eficiente desde el punto de vista económico, las obligaciones asumidas. De esta manera, aquellos que reduzcan sus emisiones en mayor medida que lo comprometido podrán vender los créditos excedentes a los Estados que consideren más difícil u oneroso satisfacer sus objetivos.

\section{Cambio climático en el Derecho de la Unión Europea}

\subsection{Instrumentos generales}

La Unión Europea asumió formalmente la problemática con el lanzamiento del Programa Europeo sobre el Cambio Climático (PECC), aprobado en el año 2000, cuyo objetivo fue permitir a todas las partes interesadas participar en los trabajos preparatorios de las políticas y medidas destinadas a reducir las emisiones de gases de efecto invernadero.

Cinco años más tarde, la Comisión Europea elaboró un documento titulado "Ganar la batalla contra el cambio climático mundial", que -fundado en un análisis de los costes y ventajas de una acción en este ámbito- estableció las bases de una futura estrategia comunitaria. En este sentido, determinó que dicha estrategia debía desplegarse en cuatro ámbitos distintos: el riesgo climático en sí mismo y la voluntad política de hacer frente al mismo, la participación internacional en la lucha contra el cambio climático, la innovación necesaria para una modificación de los métodos de producción y utilización de la energía, y la adaptación de los países a los efectos inevitables del fenómeno.

En 2007 la Unión Europea dio un nuevo paso al aprobar el instrumento denominado "Limitar el calentamiento mundial a $2^{\circ} \mathrm{C}$ - Medidas necesarias hasta 2020 y después", que propuso diversas acciones concretas para enfrentar el cambio climático y reducir la posibilidad de graves perturbaciones irreversibles a nivel planetario. Determinó asimismo como objetivo restringir la elevación media de la temperatura a $2{ }^{\circ} \mathrm{C}$ a escala mundial respecto del nivel preindustrial. 
Los avances reseñados, concordantes y con proyección futura, constituyeron muestras evidentes de la decisión de adoptar medidas para estabilizar la concentración de gases de efecto invernadero en la atmósfera (Barroso, 2008, p. 15).

\subsection{El comercio de derechos de emisión}

En materia de acciones concretas, la Unión Europea se propuso apenas iniciado el siglo XXI implementar un régimen de conformidad con el mecanismo de comercio de emisiones previsto en el Protocolo de Kioto, por medio del cual se asignaran a las empresas cuotas de emisiones en función de los objetivos ambientales de los respectivos Estados.

\subsubsection{Libro Verde}

Como primer antecedente de la política relativa al comercio de derechos de emisión se presentó, en marzo de 2000, el Libro Verde sobre el comercio de los derechos de emisión de gases de efecto invernadero en la Unión Europea (Sanz Rubiales, 2007, p. 113), cuya principal finalidad fue suscitar formalmente al debate sobre la cuestión. El documento representó el comienzo de un proceso de consulta que permitió a las partes interesadas, tanto gubernamentales como no gubernamentales, dar su parecer sobre la forma en que la Unión Europea habría de actuar para encontrar un equilibrio en el uso de los derechos de emisión.

Fue así que el año 2001 la Comisión lanzó al Consejo y al Parlamento una Propuesta de Directiva que culminó dos años más tarde con la adopción de la Directiva 2003/87.

\subsubsection{Régimen para el comercio de derechos de emisión}

\subsubsection{Eje del sistema: Directiva 2003/87}

La Directiva 2003/87 estableció el Régimen para el comercio de derechos de emisión de gases de efecto invernadero, que combina la decisión desde instancias públicas con la dinámica del mercado. Primero existe una asignación de derechos de emisión desde una racionalidad pública sobre la base de un plan; a partir de allí, pueden los titulares negociar, transmitir e intercambiar esos derechos, surgiendo una racionalidad privada que complementa y flexibiliza la asignación inicial (Esteve Pardo, 2005, pp. 253-254). 


\subsection{Conceptos fundamentales}

La Directiva gira en torno a dos conceptos clave, a saber:

- Derecho de emisión: es el título que autoriza a emitir una tonelada de dióxido de carbono, o de cualquier otro gas de efecto invernadero equivalente, durante un período determinado; es transferible.

- Permiso de emisión: es la autorización expedida por un órgano competente que debe poseer toda instalación contemplada en el Anexo I de la Directiva que realice emisiones de gases de efecto invernadero. Entre las instalaciones del Anexo I se encuentran las dedicadas a actividades energéticas, producción y transformación de metales férreos, industrias minerales, fabricación de papel y cartón. Las autoridades concederán el permiso si consideran que el titular de la instalación es capaz de garantizar el seguimiento y la notificación de las emisiones (Plaza Martín, 2005, p. 618).

\subsection{Permisos de emisión}

El requisito previo para la entrada en el mercado es la obtención de un específico permiso de emisión de gases de efecto invernadero. Como explica Esteve Pardo (2005, pp. 254-255), uno de los más destacados iusambientalistas a nivel internacional, su tramitación responde al esquema procedimental común de la fórmula autorizatoria: una solicitud por el titular de una instalación o de un proyecto de instalación, y una resolución por la Administración.

Como una variante del permiso singular, configurando un tipo con perfiles propios, se contempla el de agrupación de instalaciones. Para obtenerlo, es necesario que todas las instalaciones que pretenden integrarse dispongan ya de un permiso para la emisión de gases de efecto invernadero (Esteve Pardo, 2005, pp. 254255).

\subsection{Derechos de emisión}

\subsection{Asignación}

Las instalaciones que cuenten con un permiso pueden acceder a la titularidad de los derechos de emisión por dos vías: una es mediante la asignación originaria desde instancias estatales; la otra, a través de una reasignación adquiriéndolos en el mercado (Esteve Pardo, 2005, p. 255). 
Los instrumentos clave y puntos de partida del sistema son los Planes Nacionales de Asignación, que constituyen documentos elaborados por las autoridades de cada Estado en los que se determina la cantidad total de derechos de emisión que se va a conceder a las instalaciones del Anexo I de la Directiva, en un período anual pero de horizonte quinquenal. Según analiza Moreno Molina (2006, p. 526), una vez aprobado el Plan, se atribuye a los titulares de dichas instalaciones la cantidad de derechos prevista, con lo cual queda fijado el límite de emisiones para cada una de sus plantas.

El Plan Nacional debe determinar asimismo la cantidad de derechos reservada para nuevos entrantes, así como los criterios que regirán la distribución de dicha reserva, teniendo en cuenta el orden temporal de solicitud y el uso de tecnologías energéticamente eficientes (Esteve Pardo, 2005, p. 258).

\subsection{Entrega}

Una vez que cada titular dispone de los derechos que le hayan sido asignados para cada período, comienzan a computarse sus emisiones, pero no por la Administración sino por el propio industrial, quien al concluir cada año debe remitir a aquélla información fidedigna en la que consten las emisiones generadas en cada instalación. Para evitar fraudes, se exige que los informes sean constatados por personas u organizaciones privadas cuya profesionalidad y solvencia técnica resulten previamente acreditadas.

El titular de cada instalación debe entregar, a más tardar el 30 de abril de cada año, un número de derechos equivalente a las emisiones totales de esa instalación durante el año natural anterior, debidamente verificadas (Moreno Molina, 2006, p. 527). Quien no lo realiza en plazo, está obligado a pagar una multa de 100 euros por cada tonelada equivalente de dióxido de carbono emitido. El pago de dicha multa no exime de entregar una cantidad de derechos equivalente a la de las emisiones en exceso, llegado el momento de cumplir durante el año natural siguiente.

Un elemento novedoso que trae la Directiva es que exige a los Estados garantizar la publicación de los nombres de los infractores, lo cual constituye una medida accesoria a la penalidad que les haya sido efectivamente impuesta. 


\subsection{Mercado de derechos de emisión}

Los derechos de emisión asignados pueden ser objeto de comercio en el mercado, constituyéndose así la racionalidad privada del sistema. En efecto, son transferibles entre instalaciones de un mismo titular, entre empresas de idéntico o de diferente sector industrial, e incluso entre sujetos de la Unión Europea y titulares en terceros países en los que tales derechos sean reconocidos. Normalmente, esta transferencia se producirá del siguiente modo: el titular de una instalación que haya emitido menos toneladas de dióxido de carbono que las que le fueron asignadas tendrá un excedente de derechos sin utilizar que podrá transmitir a la empresa titular de una instalación que haya emitido más de la cuenta. Al final del año, el exceso de una se habrá compensado con el defecto de otra y la suma conjunta reflejará el mismo guarismo inicial de ambas, al principio del año (Moreno Molina, 2006, p. 529).

\subsection{Registro de derechos de emisión}

Para garantizar el funcionamiento del sistema, la Directiva contempla la creación por los Estados miembros de registros públicos en donde quede constancia de la expedición, titularidad, transferencia y cancelación de derechos de emisión, y el establecimiento de un régimen normalizado en forma de bases de datos electrónicas que facilite el seguimiento.

\subsubsection{Normas complementarias y modificatorias}

\subsection{Directiva 2004/101}

La Directiva 2004/101 modificó el esquema de comercio de derechos de emisión, permitiendo canjear créditos provenientes de los otros dos mecanismos de flexibilización del Protocolo de Kioto por derechos de emisión. Desde entonces, cada crédito procedente de AC tuvo la posibilidad de convertirse en una unidad de reducción de emisiones (URE) y cada uno derivado de MDL, en una reducción certificada de emisiones (RCE) (Rivero Fernández, 2009, p. 97). Este cambio tendió a aminorar los costes y mejorar la liquidez en el mercado europeo (Sarasíbar Iriarte, 2006, pp. 161-162).

\subsection{Directivas 2008/101 y 2009/29}

Posteriormente se aprobaron otras dos directivas que reformulan el régimen de comercio de derechos de emisión y que extienden su ámbito de aplicación: 
- La Directiva 2008/101, que incluyó las emisiones derivadas de actividades de aviación a fin de reducir el impacto en el cambio climático atribuible a este sector.

- La Directiva 2009/29, que efectuó una revisión general del sistema, de plena aplicación a partir de 2013 (Pernas García, 2009). Entre otros aportes, consagró a la subasta como el principio básico para la asignación de derechos de emisión, por considerarla el mecanismo más sencillo y eficiente desde el punto de vista económico.

\subsection{Reglamentos 219/2009 y 1031/2010}

En cuanto a los reglamentos que modificaron la Directiva 2003/87, cabe mencionar:

- El Reglamento 219/2009, que confirió competencias a la Comisión para adoptar ciertas disposiciones de aplicación para el seguimiento y la notificación de emisiones, así como normas para un régimen normalizado y garantizado de registros nacionales y para el reconocimiento de derechos en virtud de acuerdos con terceros países.

- El Reglamento 1031/2010, que estableció el calendario y reguló la gestión y las subastas de los derechos de emisión de gases de efecto invernadero.

\subsection{Decisiones $2006 / 780$ y 2007/589}

Complementaron el régimen de la Directiva 2003/87 dos Decisiones, referidas una al cómputo de las reducciones de emisiones y la otra al proceso de seguimiento y notificación.

- La Decisión 2006/780, que procuró evitar el doble cómputo de las reducciones de emisiones de gases de efecto invernadero en el régimen comunitario de comercio de derechos de emisión.

- La Decisión 2007/589, que pautó diversas directrices generales (Anexo I), así como otras suplementarias relativas al seguimiento y la notificación de emisiones (Anexos II a XI) y a los sistemas de medición continua (Anexo XII). 


\section{Conclusión}

Como corolario del presente estudio, es preciso poner de resalto como lo hicimos al inicio, que el cambio climático no constituye un problema futuro, sino una acuciante realidad. Por ello requiere acciones urgentes, a fin de limitarlo en la mayor medida posible.

En tal sentido, la Unión Europea ha otorgado prioridad a este objetivo, conforme se advierte del compromiso asumido con respecto al Protocolo de Kioto y del complejo régimen previsto por la Directiva 2003/87, sus normas modificatorias y complementarias. Sin reposar exclusivamente en disposiciones legislativas, ha diseñado un sistema que continúa adaptando y ajustando de manera constante, con el propósito de alcanzar cada vez de modo más eficiente las metas proyectadas.

El comercio de derechos de emisión se ha constituido, de conformidad con el panorama reseñado, en una herramienta fundamental e innovadora mediante la cual la Unión Europea viabiliza las acciones tendientes a mitigar los efectos adversos del cambio climático, a fin de evitar el impacto en la generación presente y las futuras.

\section{Referencias Bibliográficas}

Aguilar Rojas, G. \& Iza, A. (2009). Derecho Ambiental en Centroamérica, (T. 2.). Gland: UICN.

Allison, I., Bindoff, N., Bindschadler, R., Cox, P., de Noblet-Ducoudré, N., England, M. et al. (2009). The Copenhagen Diagnosis. Updating the World on the Latest Climate Science. Sidney: The University of New South Wales Climate Change Research Centre.

Arcocha, C. E. \& Allende Rubino, H. (2007). Tratado de Derecho Ambiental. Rosario: Nova Tesis.

Barroso, J. (2008). Sustainable Developments: Europe Leads from the Front. En J. Barroso, E. Cihelková \& Jakub Krč, M. Falkenmark, S. Faucheux, J. Figel', J. Gil-Robles et al. The European Union and World Sustainable Development. Visions of Leading Policy Makers \& Academics (p. 10-17). Luxembourg: Office for Official Publications of the European Communities.

Bellorio Clabot, D. (2004). Tratado de Derecho Ambiental, (T. 2). Buenos Aires: AdHoc. 
Camps, S. (2010, 29 de noviembre). Dura batalla para capturar las sustancias que dañan el planeta. Diario Clarín.

Cielsa, W. (1996). Cambio climático, bosques y ordenación forestal: una visión de conjunto. Estudio FAO Montes, (126), 19-23.

Comunicación de la Comisión al Consejo y al Parlamento Europeo sobre políticas y medidas de la UE para reducir las emisiones de gases de efecto invernadero: hacia un Programa Europeo sobre el Cambio Climático (PECC). EUR-Lex. Recuperado el 11 de noviembre de 2013, de http://europa.eu/legislation_summaries/environment/tackling_climate_change/128185_es.htm

Comunicación de la Comisión al Consejo, al Parlamento Europeo, al Comité Económico y Social Europeo y al Comité de las Regiones: Ganar la batalla contra el cambio climático mundial. EUR-Lex. Recuperado el 11 de noviembre de 2013, de http://europa.eu/legislation_summaries/environment/tackling_climate_change/128157_es.htm

Comunicación de la Comisión al Consejo, al Parlamento Europeo, al Comité Económico y Social Europeo y al Comité de las Regiones: Limitar el calentamiento mundial a $2{ }^{\circ} \mathrm{C}$ - Medidas necesarias hasta 2020 y después. EUR-Lex. Recuperado el 11 de noviembre de 2013, de http://eur-lex.europa.eu/LexUriServ/ LexUriServ.do?uri=COM:2007:0002:FIN:ES:PDF

Convención Marco de las Naciones Unidas sobre el Cambio Climático. United Nations Framework Convention on Climate Change. Recuperado el 11 de noviembre de 2013, de http://unfccc.int/resource/docs/convkp/convsp.pdf

Cuatecontzi, D. \& Gasca, J. (2004). Los gases regulados por la Convención Marco de las Naciones Unidas sobre el Cambio Climático. En J. Martínez \& A. Fernández Bremauntz (Comp.). Cambio climático: una visión desde México (pp. 87-98). México D.F.: Secretaría de Medio Ambiente y Recursos Naturales Instituto Nacional de Ecología.

Decisión 2006/780/CE de la Comisión, de 13 de noviembre de 2006, relativa a la forma de evitar el doble cómputo de las reducciones de emisiones de gases de efecto invernadero en el régimen comunitario de comercio de derechos de emisión en relación con actividades de proyectos derivados del Protocolo de Kioto de conformidad con la Directiva 2003/87/CE. (2006). EUR-Lex. Recuperado el 11 de noviembre de 2013, de http://eur-lex.europa.eu/LexUriServ/LexUriServ.do?uri=OJ:L:2006:316:0012:0017:ES:PDF

Decisión 2007/589/CE de la Comisión de 18 de julio de 2007 por la que se establecen directrices para el seguimiento y la notificación de las emisiones de gases de efecto invernadero de conformidad con la Directiva 2003/87/CE del Parlamento Europeo y del Consejo. (2007). EUR-Lex. Recuperado el 11 de noviembre de 2013, de http://eur-lex.europa.eu/LexUriServ/LexUriServ.do?uri=OJ:L:2007:229:0001:0085:ES:PDF 
Directiva 2003/87/CE del Parlamento Europeo y del Consejo de 13 de octubre de 2003 por la que se establece un régimen para el comercio de derechos de emisión de gases de efecto invernadero en la Comunidad y por la que se modifica la Directiva 96/61/CE del Consejo. EUR-Lex. Recuperado el 11 de noviembre de 2013, de http://eur-lex.europa.eu/LexUriServ/LexUriServ.do?uri=O$\mathrm{J}: \mathrm{L}: 2003: 275: 0032: 0046: \mathrm{es}: \mathrm{PDF}$

Directiva 2008/101/CE del Parlamento Europeo y del Consejo, de 19 de noviembre de 2008, por la que se modifica la Directiva 2003/87/CE con el fin de incluir las actividades de aviación en el régimen comunitario de comercio de derechos de emisión de gases de efecto invernadero. EUR-Lex. Recuperado el 11 de noviembre de 2013, de http://eur-lex.europa.eu/LexUriServ/LexUriServ. do?uri=OJ:L:2009:008:0003:0021:es:PDF

Directiva 2009/29/CE del Parlamento Europeo y del Consejo, de 23 de abril de 2009, por la que se modifica la Directiva 2003/87/CE para perfeccionar y ampliar el régimen comunitario de comercio de derechos de emisión de gases de efecto invernadero. (2009). EUR-Lex. Recuperado el día 11 de noviembre de 2013, de http://eur-lex.europa.eu/LexUriServ/LexUriServ.do?uri=O$\mathrm{J}: L: 2009: 140: 0063: 0087: e s: P D F$

Esteve Pardo, J. (2005). Derecho del medio ambiente. Madrid: Marcial Pons.

Juste Ruiz, J. (1999). Derecho Internacional del Medio Ambiente. (2a . ed.). Madrid: McGraw-Hill.

Kenneth Hare, F. \& Hutchinson, T. C. (1999). Perturbaciones ambientales de origen humano. En J. G. Henry \& G. W. Heinke (Dir.). Ingeniería ambiental, (pp. 111-141). (2a . ed.). México: Prentice Hall.

Kramer, F. (2003). Educación ambiental para el desarrollo sostenible. Madrid: La Catarata.

Libro Verde sobre el comercio de los derechos de emisión de gases de efecto invernadero en la Unión Europea. EUR-Lex. Recuperado el 11 de noviembre de 2013, de http://eur-lex.europa.eu/LexUriServ/site/es/com/2000/com2000_0087es01. pdf

Martínez Ataz, E. \& Díaz de Mera Morales, Y. (2004). Contaminación atmosférica. Cuenca: Universidad de Castilla-La Mancha.

Moreno Molina, Á. (2006). Derecho comunitario del medio ambiente. Marco institucional, regulación sectorial y aplicación en España. Madrid: Marcial Pons.

Moreno Sánchez, A. \& Urbina Soria, J. (2008). Impactos sociales del cambio climático en México. México: INE-PNUD.

Novelli, M. H. (2007). Ambiente, globalización e integración. El Derecho Ambiental en el Mercosur. En C. E. Arcocha \& H. L. Allende Rubino, Tratado de Derecho Ambiental (pp. 203-234). Rosario: Nova Tesis.

Pachauri, R. \& Reisinger, A. (Dir.) (2008). Cambio climático 2007: Informe de síntesis. Ginebra: Grupo Intergubernamental de Expertos sobre el Cambio Climático. 
Pernas García, J. (2009). Actualidad. Borradores de anteproyecto en materia de comercio de emisiones y almacenamiento geológico. Actualidad Jurídica Ambiental. Recuperado el 30 de julio de 2013, de http://www.actualidadjuridicaambiental. $\mathrm{com} / \mathrm{?} \mathrm{p}=2004$

Petit, J. \& Prudent, G. (2008). Cambio Climático y Biodiversidad en los Territorios de Ultramar de la Unión Europea. Gland - Bruselas: UICN.

Plaza Martín, C. (2005). Derecho Ambiental de la Unión Europea. Valencia: Tirant lo Blanch.

Protocolo de Kyoto de la Convención Marco de las Naciones Unidas sobre el Cambio Climático. United Nations Framework Convention on Climate Change. Recuperado el 11 de noviembre de 2013, de http://unfccc.int/resource/docs/ convkp/kpspan.pdf

Reglamento (CE) no 219/2009 del Parlamento Europeo y del Consejo de 11 de marzo de 2009 por el que se adaptan a la Decisión 1999/468/CE del Consejo determinados actos sujetos al procedimiento establecido en el artículo 251 del Tratado, en lo que se refiere al procedimiento de reglamentación con control. EUR-Lex. Recuperado el 11 de noviembre de 2013, de http://eur-lex.europa. eu/LexUriServ/LexUriServ.do?uri=OJ:L:2009:087:0109:0109:ES:PDF

Reglamento (UE) n 1210/2011 de la Comisión, de 23 de noviembre de 2011, por el que se modifica el Reglamento (UE) no 1031/2010, en particular con el fin de determinar el volumen de los derechos de emisión de gases de efecto invernadero por subastar antes de 2013. (2011). EUR-Lex. Recuperado el 11 de noviembre de 2013, de http://eur-lex.europa.eu/LexUriServ/LexUriServ. do?uri=OJ:L:2011:308:0002:01:ES:HTML

Reglamento (UE) no 1031/2010 de la Comisión de 12 de noviembre de 2010 sobre el calendario, la gestión y otros aspectos de las subastas de los derechos de emisión de gases de efecto invernadero con arreglo a la Directiva 2003/87/CE del Parlamento Europeo y del Consejo, por la que se establece un régimen para el comercio de derechos de emisión de gases de efecto invernadero en la Comunidad. EUR-Lex. Recuperado el 11 de noviembre de 2013, de http://eur-lex.europa.eu/LexUriServ/LexUriServ.do?uri=OJ:L:2010:302:0001:01:ES:HTML

Reglamento (UE) no 784/2012 de la Comisión, de 30 de agosto de 2012, por el que se modifica el Reglamento (UE) no 1031/2010 para registrar una plataforma de subastas que va a designar Alemania y se corrige su artículo 59, apartado 7 Texto pertinente a efectos del EEE. EUR-Lex. Recuperado el 11 de noviembre de 2013 de, http://eur-lex.europa.eu/LexUriServ/LexUriServ.do?uri=OJ:L:2010:302:0001:01:ES:HTML 
Reglamento (UE) No 920/2010 de la Comisión, de 7 de octubre de 2010, relativo a un sistema normalizado y garantizado de registros de conformidad con la $\mathrm{Di}$ rectiva 2003/87/CE del Parlamento Europeo y del Consejo y la Decisión no 280/2004/CE del Parlamento Europeo y del Consejo. EUR-Lex. Recuperado el 11 de noviembre de 2013, de http://eur-lex.europa.eu/LexUriServ/LexUriServ.do?uri=OJ:L:2010:270:0001:0052:ES:PDF

Rivero Fernández, C. (2009). Guía sobre el comercio de derechos de emisión de gases de efecto invernadero. Alcalá de Henares: Asociación de Empresarios del Henares - Fundación Mapfre.

Sanz Rubiales, Í. (2007). El mercado de derechos a contaminar: régimen jurídico-público del mercado comunitario de derechos de emisión en España, (T. 2). Valladolid: Lex Nova.

Sarasíbar Iriarte, M. (2006). Régimen jurídico del cambio climático. Valladolid: Lex Nova.

Saura Estapà, J. (2003). El cumplimiento del Protocolo de Kioto sobre cambio climático. Barcelona: Universitat de Barcelona.

Torres, J. \& Gómez, A. (2008). Adaptación al cambio climático: de los fríos y los calores en los Andes. Lima: Soluciones prácticas-ITDG. 